\title{
Poker Flat Incoherent Scatter Radar observations of anomalous electron heating in the $\mathrm{E}$ region
}

\author{
R. A. Makarevich ${ }^{1}$, A. V. Koustov ${ }^{2}$, and M. J. Nicolls ${ }^{3}$ \\ ${ }^{1}$ Geophysical Institute and Department of Physics, University of Alaska Fairbanks, Fairbanks, AK, USA \\ ${ }^{2}$ Institute of Space and Atmospheric Studies, University of Saskatchewan, Saskatoon, SK, Canada \\ ${ }^{3}$ Center for Geospace Studies, SRI International, Menlo Park, CA, USA \\ Correspondence to: R. A. Makarevich (r.makarevich@gi.alaska.edu)
}

Received: 6 March 2013 - Revised: 22 May 2013 - Accepted: 30 May 2013 - Published: 3 July 2013

\begin{abstract}
A comprehensive 2-year dataset collected with the Poker Flat Incoherent Scatter Radar (PFISR) located near Fairbanks, Alaska (MLAT $=65.4^{\circ} \mathrm{N}$ ) is employed to identify and analyse 22 events of anomalous electron heating (AEH) in the auroral $\mathrm{E}$ region. The overall AEH occurrence probability is conservatively estimated to be $0.3 \%$ from nearlycontinuous observations of the $\mathrm{E}$ region by PFISR, although it increases to $0.7-0.9 \%$ in the dawn and dusk sectors where all AEH events were observed. The AEH occurrence variation with MLT is broadly consistent with those of events with high convection velocity $\left(>1000 \mathrm{~m} \mathrm{~s}^{-1}\right)$ or electron temperature $(>800 \mathrm{~K})$, except for much smaller AEH probability and absence of AEH events near magnetic midnight. This suggests that high convection electric field by itself is necessary but not sufficient for measurable electron heating by two-stream plasma waves. The multi-point observations are utilised to investigate the fundamental dependence of the electron temperature on the convection electric field, focusing on the previously-proposed saturation effects at extreme electric fields. The AEH dataset was found to exhibit considerable scatter and, on average, similar rate of the electron temperature increase with the electric field up to $100 \mathrm{mV} \mathrm{m}^{-1}$ as compared with previous studies. At higher (highest) electric fields, the electron temperatures are below the linear trend on average (within uncertainty). By employing a simple fluid model of AEH, it is demonstrated that some of this deviation from the linear trend may be due to a stronger vibrational cooling at very large temperatures and electric fields.
\end{abstract}

Keywords. Ionosphere (Auroral ionosphere; plasma waves and instabilities; wave-particle interactions)

\section{Introduction}

Anomalous electron heating or AEH represents an important example of how small-scale plasma instability processes can impact directly and dramatically ionospheric properties. AEH has been first discovered using an incoherent scatter radar (ISR) at Chatanika, Alaska as strong enhancement of the electron temperature $T_{\mathrm{e}}$ in the high-latitude $\mathrm{E}$ region (Schlegel and St.-Maurice, 1981; St.-Maurice et al., 1981; Wickwar et al., 1981). It has been since observed by other ISR facilities including the European Incoherent Scatter (EISCAT) system (Igarashi and Schlegel, 1987; Williams et al., 1992; St.-Maurice et al., 1999), the Millstone Hill ISR (Foster and Erickson, 2000), and the Sondrestrom ISR (Milikh et al., 2006; Bahcivan, 2007). AEH has also been observed by coherent auroral radars as strong enhancements in the irregularity phase velocity assumed to be related to $T_{\mathrm{e}}$ via its dependence on the ion-acoustic speed (e.g. Nielsen and Schlegel, 1985; Makarevich, 2008) and by riometers in the polar cap as elevated cosmic noise absorption related to $T_{\mathrm{e}}$ via collision frequency (Stauning, 1984; Stauning and Olesen, 1989).

The electron temperature enhancements were found to correlate with the convection electric field $\boldsymbol{E}$ or the $\boldsymbol{E} \times \boldsymbol{B}$ convection velocity $V_{E}$ and exhibit an approximately linear increase of $T_{\mathrm{e}}$ with $V_{E}$ for sufficiently large drifts $V_{E}>$ $600 \mathrm{~m} \mathrm{~s}^{-1}$ (Williams et al., 1992). It was clear right from the start though that the electron temperature increase was too strong to be accounted by the Joule or particle heating alone and a mechanism involving electron heating by unstable waves was proposed (St.-Maurice et al., 1981). The measured electron temperature relationship with the electric field 
was successfully reproduced for large convection drifts using both kinetic (St.-Maurice and Schlegel, 1982; Robinson and Honary, 1990) and fluid (Robinson, 1986; Robinson and Honary, 1993) models of AEH. Most of these earlier models invoked anomalous diffusion processes (Sudan, 1983) occurring during development of the modified two-stream instability (MTSI), also known as the Farley-Buneman instability. It has also been realised that perpendicular anomalous wave heating cannot be solely responsible for all of the electron temperature increase (e.g. Janhunen, 1994) and that parallel electric fields must be involved (St.-Maurice and Laher, 1985). More recently, the AEH models have been extended to include effects of turbulent electric fields and nonMaxwellian electron distribution (Dimant and Milikh, 2003; Milikh and Dimant, 2003; Bahcivan et al., 2006; Dimant and Oppenheim, 2011a; Oppenheim and Dimant, 2013) with numerical modelling results showing a somewhat steeper increase of $T_{\mathrm{e}}$ with $E$ (Milikh and Dimant, 2003).

Experimental studies focused on analysis of individual $\mathrm{AEH}$ events that were found during strong geomagnetic disturbances (Schlegel and St.-Maurice, 1981; Milikh et al., 2006; Bahcivan, 2007) and were associated with either wide regions of enhanced electric fields or narrow convection channels in the vicinity of auroral arcs (Williams et al., 1992). The approach in deriving electric fields included beam-swinging technique using multi-point ion drift measurements $V_{\mathrm{i}}$ (Schlegel and St.-Maurice, 1981) and tri-static ion drift measurements (Williams et al., 1992) as well as proxy $E$ estimates from coherent backscatter power (Foster and Erickson, 2000) and the F region ion temperature $T_{\mathrm{i}}$ (St.Maurice et al., 1999), or some combination of those (Bahcivan, 2007). Even though AEH has been long accepted as a fact of life in high-latitude ionospheric phenomenology (St.Maurice et al., 1999), it is still largely unknown what local conditions are conducive to its occurrence, apart from enhanced convection, or even how often AEH occurs.

The electric fields $E$ at which the electron temperature exhibits a linear relationship with $E$ have been shown to be generally between $30-120 \mathrm{mV} \mathrm{m}^{-1}$, with larger $E$ values very rarely observed. An interesting issue raised recently is a possible departure from this linearity or even saturation in the MTSI wave heating at extreme electric fields $E>$ $120 \mathrm{mV} \mathrm{m}^{-1}$ (Bahcivan, 2007). Even though no evidence for this effect has been found so far, the potential importance of this effect to modelling efforts makes further investigations into the fundamental relationship between $T_{\mathrm{e}}$ and $E$ a useful effort, particularly utilising the largest possible extent of the electric field.

A related issue in this context is how the signal-to-noise ratio (SNR) measured by an ISR system and inferred electron density $N_{\mathrm{e}}$ depends on the electron temperature $T_{\mathrm{e}}$ and the electric field $E$. In addition to the two well-known effects of (1) SNR depending on $T_{\mathrm{e}}$ and (2) $N_{\mathrm{e}}$ anti-correlating with $E$ near precipitation regions, enhanced $T_{\mathrm{e}}$ may reduce recom- bination rates (Schlegel, 1982) causing a gradual increase in $N_{\mathrm{e}}$ (Milikh et al., 2006).

The deployment of the Poker Flat Incoherent Scatter Radar (PFISR) system near Fairbanks, Alaska provided additional opportunities to re-examine AEH processes as described below. First, PFISR is capable of providing nearly-continuous measurements of the electron temperature in the $\mathrm{E}$ region with reasonable temporal resolution of 2-5 min, which allows to estimate the overall AEH occurrence probability and examine AEH occurrence trends. Second, the temperature measurements are routinely conducted in conjunction with the electric field measurements with good spatial resolution in MLAT of $0.25^{\circ}$, which allows to re-examine the fundamental $T_{\mathrm{e}}$-vs. $-E$ relationship using a new extensive dataset. Third, the Advanced Modular design of PFISR allows quick electronic beam-steering so that the electron temperature can be measured at several beam positions quasi-simultaneously, which enables 2-D and 3-D imaging of the local ionosphere (Semeter et al., 2009). For the purposes of the AEH study, it means that electron heating processes near the $\mathrm{E}$ region peak can be examined in 2-D context and spatial and temporal variations can potentially be separated.

The main aim of the current study is to investigate anomalous electron heating in the auroral $\mathrm{E}$ region utilising nearlycontinuous and coincident observations of the electron temperature and the convection electric field by PFISR. The specific objectives are: (1) to estimate the overall AEH occurrence probability and analyse AEH occurrence trends, and (2) to re-examine the fundamental relationship between the electron temperature and the convection electric field using the Advanced Modular ISR system, focusing on departures from the expected linear trend in this relationship.

\section{Experiment description}

The ISR dataset employed in this study comprised the data from the PFISR system located at the Poker Flat Research Range $\left(65.13^{\circ} \mathrm{N}, 147.47^{\circ} \mathrm{W}, \mathrm{MLAT}=65.4^{\circ} \mathrm{N}\right)$ near Fairbanks, Alaska (Heinselman and Nicolls, 2008). PFISR is a phased-array system with narrow beam that can be steered electronically in many directions within the viewing area. Data from multiple beams can be collected nearly simultaneously (sequentially on a pulse-by-pulse basis, but with a very high time cadence) within the same integration period. PFISR has been in routine operation since 1 March 2007 and the current study considered the data in 2010-2011. This was the period with the most recent processed data with nearlycontinuous yearly coverage, predominantly high temporal resolution of 3-5 min, and significant geomagnetic activity.

In this study, only the data from the two following series of PFISR modes were employed: (1) the International Polar Year (IPY) mode data collected using low-duty cycle (Sojka et al., 2009) and (2) the Time History of Events and Macroscale Interactions during Substorms (THEMIS) mode 

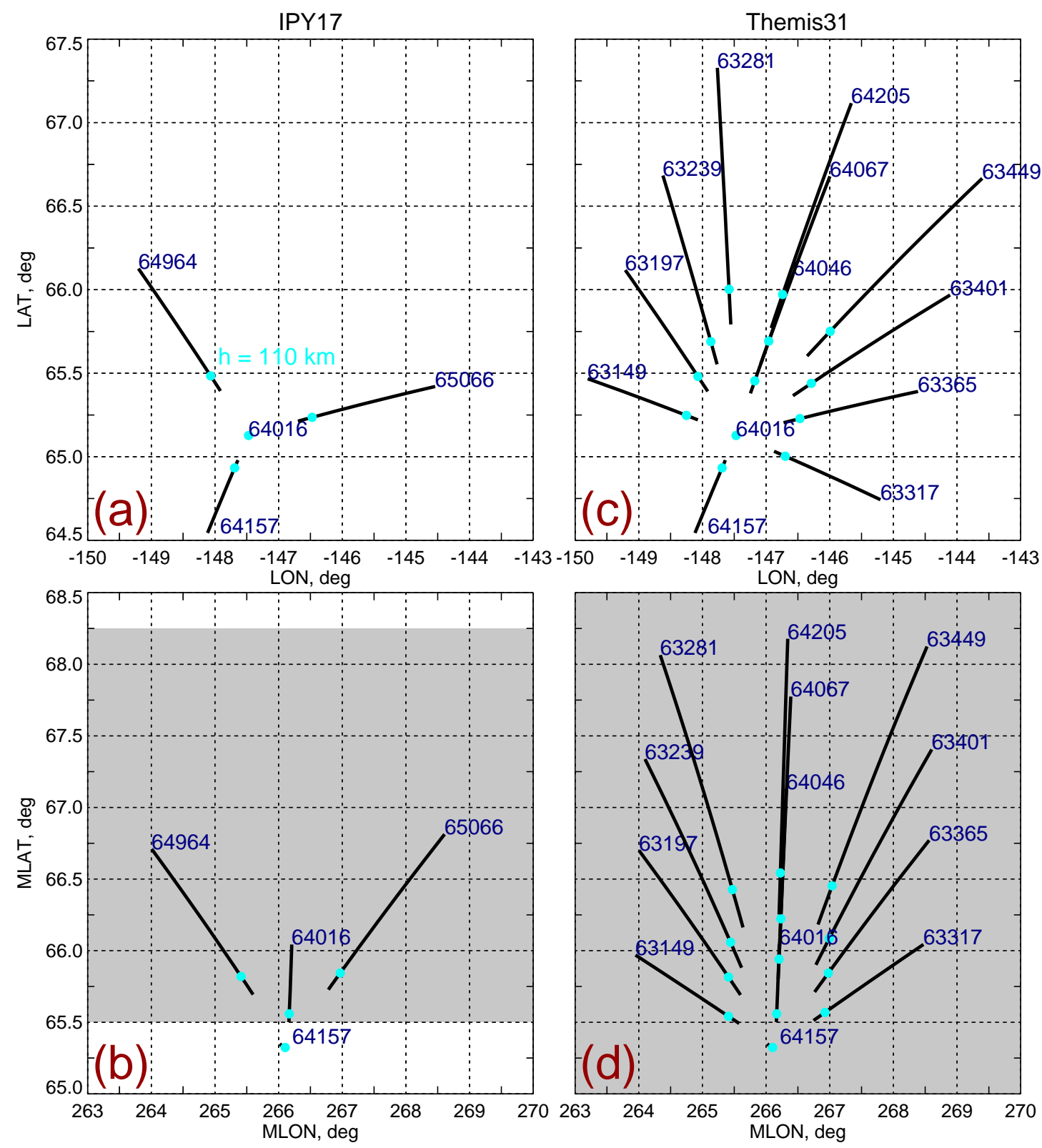

Fig. 1. PFISR experiment configuration for (a) and (b) IPY17 and (c) and (d) Themis 31 modes. Shown are the PFISR beams in (a) and (c) geographic and (b) and (d) geomagnetic coordinates corresponding to the alternative coding (AC) data. The start of the gate nearest to a $110-\mathrm{km}$ altitude is shown by a circle for each beam. The standard PFISR beam identification is also given by the digits near the maximum altitude coordinates. The grey-shaded area in (b) and (d) shows magnetic latitudes with convection ion velocity and electric field vectors from the long pulse (LP) data.

data used for substorm studies (Lyons et al., 2009). The former included only the IPY17 variation of the IPY mode and the latter included variations THEMIS30 and THEMIS31, as well as Lyons30, a slight modification of THEMIS30. Figure 1 shows representative footprints of the PFISR beams in both series in (a) and (c) geographic and (b) and (d) geomagnetic coordinates. The standard PFISR beam numbers are shown by the digits near each footprint. The IPY (THEMIS)
PFISR data were collected in 4 (13) beams, as illustrated in Fig. 1.

Both modes used 2 sets of interleaved pulses: a long pulse (LP) with $72 \mathrm{~km}$ range resolution designed for $F$ region studies and an alternating code (AC) pulse with $4.5 \mathrm{~km}$ resolution used for E region studies. In this study, we employed the AC data for the electron temperature and density products near $110 \mathrm{~km}$. Figure 1 shows footprints corresponding to the AC 

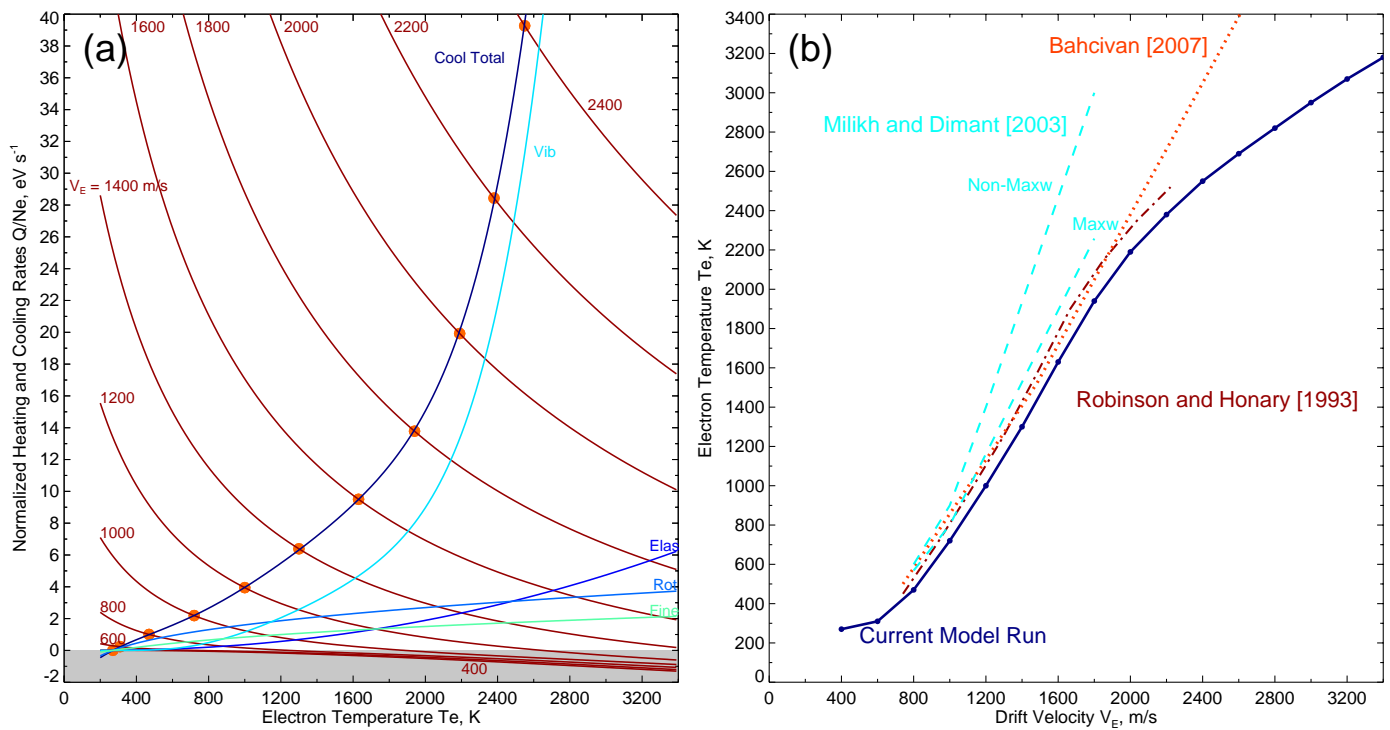

Fig. 2. (a) Normalised heating and cooling rates versus the electron temperature. Red lines are the heating rate function $Q$ divided by the electron density $N_{\mathrm{e}}$ for different values of the convection drift speed $V_{E}$. The blue and green lines are (from top to bottom): total cooling rate, cooling due to vibrational excitation of $\mathrm{N}_{2}$ and $\mathrm{O}_{2}$, cooling due to elastic electron-neutral interactions, cooling due to rotational excitation of $\mathrm{N}_{2}$ and $\mathrm{O}_{2}$, and cooling due to fine structure excitation of $\mathrm{O}$. Red circles indicate balance temperatures between heating and cooling. (b) Various model dependencies of electron temperature versus convection drift velocity. The solid line shows the results of the current fluid model from panel (a). Other selected model results are also shown as indicated in Fig. 2b and explained in the text.

data only, and the range gate nearest to $110 \mathrm{~km}$ is shown by a blue circle for each beam.

In the present study, the standard electric field data corrected for the neutral motions were also employed. These estimates were obtained from the line-of-sight ion velocity LP data using a method described by Heinselman and Nicolls (2008). The electric field data were available in the greyshaded areas in MLAT in Fig. 1b and d. The 3-D electric field vectors are obtained from the ion drift components in LP gates above $150 \mathrm{~km}$ in altitude by fitting and binning in MLAT; these estimates are most accurate close to the "middle" beams $(64 \mathrm{xxx})$ or near MLON of $266.25^{\circ} \mathrm{E}$. For the occurrence part of the current study, we employed the standard data products at $15 \mathrm{~min}$ (IPY in 2010), $5 \mathrm{~min}$ (IPY in 2011), and 3 min (THEMIS), because re-analysis of the entire 2-year PFISR dataset at a better time resolution was not feasible. For all identified AEH events however, the PFISR data were re-analysed at a 2 min time resolution and with minimum assumptions in the ISR spectrum fitting (e.g. without assuming $T_{\mathrm{e}}=T_{\mathrm{i}}$ ). The focus of other parts of the current study was thus solely on the higher-time-resolution data at $2 \mathrm{~min}$ integration, Sect. 5. For further details on technical specifications of the PFISR system the reader is referred to Heinselman and Nicolls (2008).

\section{Anomalous electron heating models}

Several past experimental studies reported on the linear relationship between the electron temperature and the convection electric field above the MTSI threshold of $20-30 \mathrm{mV} \mathrm{m}^{-1}$ (e.g. Williams et al., 1992; Bahcivan, 2007). While theoretical efforts were generally in excellent agreement with these results, matching closely the experimentally-obtained trends of $T_{\mathrm{e}}$-vs.- $E$, very few of those have considered extreme electric field values of $>100 \mathrm{mV} \mathrm{m}^{-1}$ (or convection drifts $V_{E}$ of $>2000 \mathrm{~m} \mathrm{~s}^{-1}$ ). Robinson and Honary (1993) considered drifts $V_{E}$ of up to $2400 \mathrm{~m} \mathrm{~s}^{-1}\left(E=120 \mathrm{mV} \mathrm{m}^{-1}\right)$ and their results based on the fluid formalism did show a somewhat slower $T_{\mathrm{e}}$ increase at $110 \mathrm{~km}$ at the highest values of $V_{E}=2000-2400 \mathrm{~m} \mathrm{~s}^{-1}$ (see their Fig. 2). In order to provide a meaningful theoretical framework in which to interpret our observations and theoretically investigate whether fluid formalism can produce a considerable deviation from the linear trend, in this section we aim to reproduce and extend their calculations to even higher convection drifts.

Figure 2a illustrates the method employed. The normalised cooling rates $Q / N_{\mathrm{e}}$ were calculated for different values of electron temperatures $T_{\mathrm{e}}$ and these are shown by blue and green curves in Fig. 2a. In finding these rates we used standard expressions for the various cooling rates given by Schunk and Nagy (1978) and neutral densities obtained from the MSISE model run for this location (Hedin, 1991). The model neutral densities were corrected by a factor of 1.43 to yield the same ion collision frequency $v_{\text {in }}=870 \mathrm{~s}^{-1}$ as that 
used by Robinson and Honary (1993) in their calculations at $110 \mathrm{~km}$. In using the corrected neutral densities we followed St.-Maurice et al. (1981) who employed a very similar correction method. From Fig. 2a, the cooling due to vibrational excitation of $\mathrm{N}_{2}$ and $\mathrm{O}_{2}$ dominates in the total cooling rate and its rate of increase changes quite dramatically between $T_{\mathrm{e}}=1800-2000 \mathrm{~K}$.

The heating rates for various drifts $V_{E}$ were also calculated using the method described by Robinson and Honary (1993) based on earlier works by Robinson (1986) and Sudan (1983). In addition to the above $v_{\text {in }}$ value, in these calculations we also used the electron collision frequencies $v_{\mathrm{en}}$ obtained from expressions given by Schunk and Nagy (1980) with the same model neutral densities as those used in the cooling rate calculations. Here we included both the normal frictional heating and anomalous heating effects, represented by the first and last terms, respectively, on the right-hand side of Eq. (9) from Robinson and Honary (1993).

The obtained normalised heating rates are shown by the red curves in Fig. 2a. The heating rates increase with $V_{E}$ but decrease with $T_{\mathrm{e}}$; this is mostly because the anomalous heating rate is proportional to $\left(V_{E}-C_{\mathrm{s}}\right)^{2}$, where the ion-acoustic speed $C_{\mathrm{s}}=\sqrt{\left(T_{\mathrm{e}}+T_{\mathrm{i}}\right) / m_{\mathrm{i}}}$. The heating rates are negative for the smallest $V_{E}$ values starting from a certain $T_{\mathrm{e}}$; this is simply a consequence of this AEH model not being able to yield large enough anomalous heating at these relatively small drifts. This artefact of the method had no effect on our calculations since all balance temperatures were obtained at positive heating values.

The electron temperatures that resulted in the balance of the heating and cooling rates were found for each drift velocity and these are shown by the red circles in Fig. 2a. The same points are shown in Fig. $2 b$ as the solid blue curve. Also shown are model trends taken from three other studies as indicated on the diagram. There is some difference between the current model trend and the trends taken from Robinson and Honary (1993) and Bahcivan (2007), but this is entirely due to a different magnetic field magnitude $B$ used in our calculations at MLAT $=65^{\circ}\left(5.4 \times 10^{-5} \mathrm{~T}\right.$ vs. $\left.5.0 \times 10^{-5} \mathrm{~T}\right)$. This is because heating rates depend on the ion gyrofrequency $\Omega_{\mathrm{i}}$ (or, more specifically, on the ratio $v_{\mathrm{in}} / \Omega_{\mathrm{i}}$ ) even though cooling rates are independent of $B$. The same modelling conducted with $B=5.0 \times 10^{-5} \mathrm{~T}$ shows a perfect match of the 3 models at $V_{E}=400-1800 \mathrm{~m} \mathrm{~s}^{-1}$.

One can see that the current model trend is approximately linear between $800-1800 \mathrm{~m} \mathrm{~s}^{-1}$. However, at larger drift speeds $V_{E}>1800 \mathrm{~m} \mathrm{~s}^{-1}$, the rates of $T_{\mathrm{e}}$ increase with $V_{E}$ become significantly smaller so that the solid curve deviates from the linear trend. The reason for this change in the rates of increase is the earlier-noted change in the cooling due to vibrational excitation.

Finally, two trends are shown for the model by Milikh and Dimant (2003). The upper (lower) dashed line is for nonMaxwellian (Maxwellian) electron distribution. The upper trend yields a generally higher rate of increase than any other model trend. This is due to an introduced correction required for the non-Maxwellian case. For the Maxwellian case, the dashed trend is consistent with other models. One should also note that the fitted PFISR products including $T_{\mathrm{e}}$ use a spectrum model assuming a Maxwellian distribution and that the AEH model by Milikh and Dimant (2003) conveniently accounts for this by computing effective electron temperatures. It is expected that the PFISR-measured $T_{\mathrm{e}}$ will be somewhere between these two trends depending on electron distribution.

The presented extension of the previously-developed AEH model towards higher drift velocities provides a useful framework for comparing with observations. The AEH model produced significantly smaller rates of increase at larger drift values, with the model curve deviating from the linear trend derived from moderate $V_{E}$ values. This modelling result is somewhat unexpected considering that most other studies were in agreement with the linear trend. It was consistent however, with the hint of a similar effect observed in the modelling results reported by Robinson and Honary (1993).

\section{Occurrence of electron heating events}

In this section, the statistical results on the AEH occurrence trends are presented starting with a description of the method used to identify AEH events. The PFISR data collected in the field-aligned beam 64157 were analysed to produce the number of AC data points within each hourly interval within the considered gate of the $110 \mathrm{~km}$ altitude. Next, for all daily intervals all "high- $T_{\mathrm{e}}$ " points at $110 \mathrm{~km}$ were identified and counted. These were defined as AC data points with $T_{\mathrm{e}} \geq$ $800 \mathrm{~K}$. A number of high- $T_{\mathrm{e}}$ points in a sequence was also counted.

An AEH event was defined as a period with (1) the total number of high- $T_{\mathrm{e}}$ points exceeding a certain critical number $n_{\mathrm{cr}}$ dependent on time resolution, (2) the total number of sequential high- $T_{\mathrm{e}}$ points being greater or equal than a certain critical number $n_{\text {seq }}$, (3) median time between high- $T_{\mathrm{e}}$ points not exceeding a critical value $t_{\mathrm{cr}}$, and (4) the total duration of an event not exceeding $6 \mathrm{~h}$. The 3 critical values adopted were: 2, 2, and $20 \mathrm{~min}$ for IPY17 data at $15 \mathrm{~min}$ resolution (in year 2010); 5, 3, and $15 \mathrm{~min}$ for IPY17 data at $5 \mathrm{~min}$ (in year 2011); and 3, 2, and $10 \mathrm{~min}$ for all THEMIS data. The different selection for the 3 critical numbers were needed because of the different time resolutions. In all cases, the identified events were "true" AEH events, that is high- $T_{\mathrm{e}}$ measurements occurring in sequences, rather than spurious $T_{\mathrm{e}}$ enhancements that were separated in time and observed over an extended period. This was verified by manually examining all daily plots of the electron and ion temperatures and convection electric fields during the 22 unique AEH events. In all 22 events, the $T_{\mathrm{e}}$ enhancements were observed in other range gates within the $\mathrm{E}$ region (not just at $110 \mathrm{~km}$ ). They 
were also accompanied by the $T_{\mathrm{i}}$ enhancements in the $\mathrm{F}$ region and strong electric fields at the MLATs of interest.

Figure 3 illustrates 2 examples of AEH events found. It shows the PFISR data collected in the field-aligned beam 64157, Fig. 1. The AEH interval (defined as described above) is shown by the blue bar and black vertical lines. In both top panels it encompasses the $T_{\mathrm{e}}$ enhancements at $110 \mathrm{~km}$ (white horizontal line) as well as associated $T_{\mathrm{e}}$ enhancements at other heights. The pink line shows the time variation of $T_{\mathrm{e}}$ at $110 \mathrm{~km}$ in arbitrary scale which is the same for both events. The maximum value in $\mathrm{K}$ is given near the peak of each line.

Both events were accompanied by strong convection velocity, as shown in the middle-row panels. The convection drifts were estimated as described in Sect. 2; they were in excess of $1000 \mathrm{~m} \mathrm{~s}^{-1}$ during the entire AEH interval for the 1st event (these periods are shown by the red bars at the bottom of middle-row panels) and for a significant fraction of the 2nd event interval roughly coinciding with the times of peak $T_{\mathrm{e}}$. The time variation of $V_{E}$ at $66.0^{\circ}$ is shown by the pink line in arbitrary scale. This was the first MLAT bin with consistently good $V_{E}$ data and even though the MLAT of $T_{\mathrm{e}}$ measurements was somewhat smaller $\left(\sim 65.3^{\circ}\right.$, Fig. $\left.1 b\right)$, the correlation analysis presented in Sect. 5 showed that this was the best possible conjunction between the $T_{\mathrm{e}}$ and $V_{E}$ measurements in this mode. As such, the time evolutions of $T_{\mathrm{e}}$ and $V_{E}$ were quite similar, with the main $T_{\mathrm{e}}$ and $V_{E}$ peaks observed at the same time.

The bottom-row panels show the electron density $N_{\mathrm{e}}$ in the same format as the electron temperature plots. The 1st event shows a deep drop in $N_{\mathrm{e}}$ accompanying the $T_{\mathrm{e}}$ and $E$ enhancements, a clear example of the expected anti-correlation between $N_{\mathrm{e}}$ and $E$. In the 2nd event, a drop in $N_{\mathrm{e}}$ was also observed, but here the $T_{\mathrm{e}}$ peaks were observed at the beginning ( $\sim 16: 00 \mathrm{UT})$ and at the end $(\sim 17: 00 \mathrm{UT})$ of the interval with depleted density. In both events, the strongest $T_{\mathrm{e}}$ enhancements were observed between the regions of enhanced density which, in this time sector, were likely to be caused by strong electron precipitation. From Fig. 3, there appears to be a positive correlation observed between $T_{\mathrm{e}}$ and $V_{E}$ and negative correlations between $T_{\mathrm{e}}$ and $N_{\mathrm{e}}$ and between $V_{E}$ and $N_{\mathrm{e}}$.

Figure $4 \mathrm{a}$ and $\mathrm{b}$ shows the time periods covered by PFISR in either IPY or THEMIS mode by the yellow stripes. The total percentages covered were $45 \%$ and $30 \%$ in 2010 in the IPY and THEMIS modes, respectively. The overall coverage was $74 \%$ in 2010 and $75 \%$ in 2011. Figure $4 \mathrm{c}$ and d presents a similar analysis but in UT (with both modes combined). These diagrams show that the coverage by PFISR was approximately uniform both in season and in UT. There was a small bias towards daytime observations in 2011, but nighttime coverage was still in excess of $60 \%$. The only 2 gaps of more than 10 days were in 7-20 July 2010 (PFISR maintenance period) and in 1-11 August 2011 (D region focussed runs).
All 22 AEH events are shown by the diamonds (IPY) and asterisks (THEMIS) in Fig. 4a and b and by the colour bars in Fig. 4c and d. The enhanced convection drift periods $\left(V_{E}>1000 \mathrm{~m} \mathrm{~s}^{-1}\right)$ close in time to AEH events are also shown by the red bars in Fig. $4 \mathrm{c}$ and d. The AEH occurrence probability is shown in the top of Fig. $4 \mathrm{c}$ and d separately for the IPY and THEMIS modes and for all modes combined. The probability was calculated as the total number of hours within AEH events divided by the total number of hours when PFISR operated either in the IPY or THEMIS mode.

The overall rate of AEH occurrence was $<0.3 \%$. The AEH events were only observed near equinoxes and at 00:00-08:00 UT or 14:00-20:00 UT (13:00-21:00 MLT or 02:00-09:00 MLT). The distribution was fairly symmetric with respect to 00:00 MLT in 2011. In 2010 however, all AEH events found were in the evening sector, which is a puzzling discrepancy. One factor is lower solar and geomagnetic activity that was observed throughout 2010; the other factor is well-known differences in morphology between eastward (pre-midnight) and westward (post-midnight) electrojets and associated $\mathrm{E}$ region wave activity (e.g. review by Fejer and Kelley, 1980).

All AEH events were associated with the periods of enhanced convection shown by the red bars in Fig. $4 \mathrm{c}$ and d, as expected. Interestingly though, the occurrence of enhanced convection $V_{E}>1000 \mathrm{~m} \mathrm{~s}^{-1}$ appears to be a necessary condition but not sufficient, as in many cases red bars started earlier than blue or green bars; the same feature was also observed in Fig. 3 for a small sample of events. One should also bear in mind that in this presentation the blue and green bars indicate AEH intervals from start to finish rather than actual $T_{\mathrm{e}}$ enhancements which may refer to a smaller fraction of the AEH interval (e.g. event 20110926). This is in contrast with the red bars of enhanced convection that show actual $V_{E}$ enhancements. This further strengthens the above argument about enhanced convection being only a necessary condition.

The value of $V_{E}=1000 \mathrm{~m} \mathrm{~s}^{-1}$ is well above both the MTSI threshold velocity and the minimum drift velocity at which AEH was observed in the past (e.g. Williams et al., 1992). In fact, this drift value closely corresponds to the $T_{\mathrm{e}}=$ $800 \mathrm{~K}$ value from Fig. $2 \mathrm{~b}$ which was selected for AEH identification in the current study. For this reason, one generally expects the periods with $T_{\mathrm{e}}>800 \mathrm{~K}$ and $V_{E}>1000 \mathrm{~m} \mathrm{~s}^{-1}$ to start at approximately the same time for exactly coincident $T_{\mathrm{e}}$ and $V_{E}$ data. The absence of exact spatial match between the $T_{\mathrm{e}}$ and $V_{E}$ data may be responsible for some temporal shifts between the high- $T_{\mathrm{e}}$ and high- $V_{E}$ periods, but one should still see a general agreement. The events showing a $T_{\mathrm{e}}$ increase well within the period of high $V_{E}$ in both Figs. 3 and 4 may therefore indicate that some other condition(s) must be satisfied for $T_{\mathrm{e}}$ to increase in line with the expected model trend illustrated in Fig. 2b. 

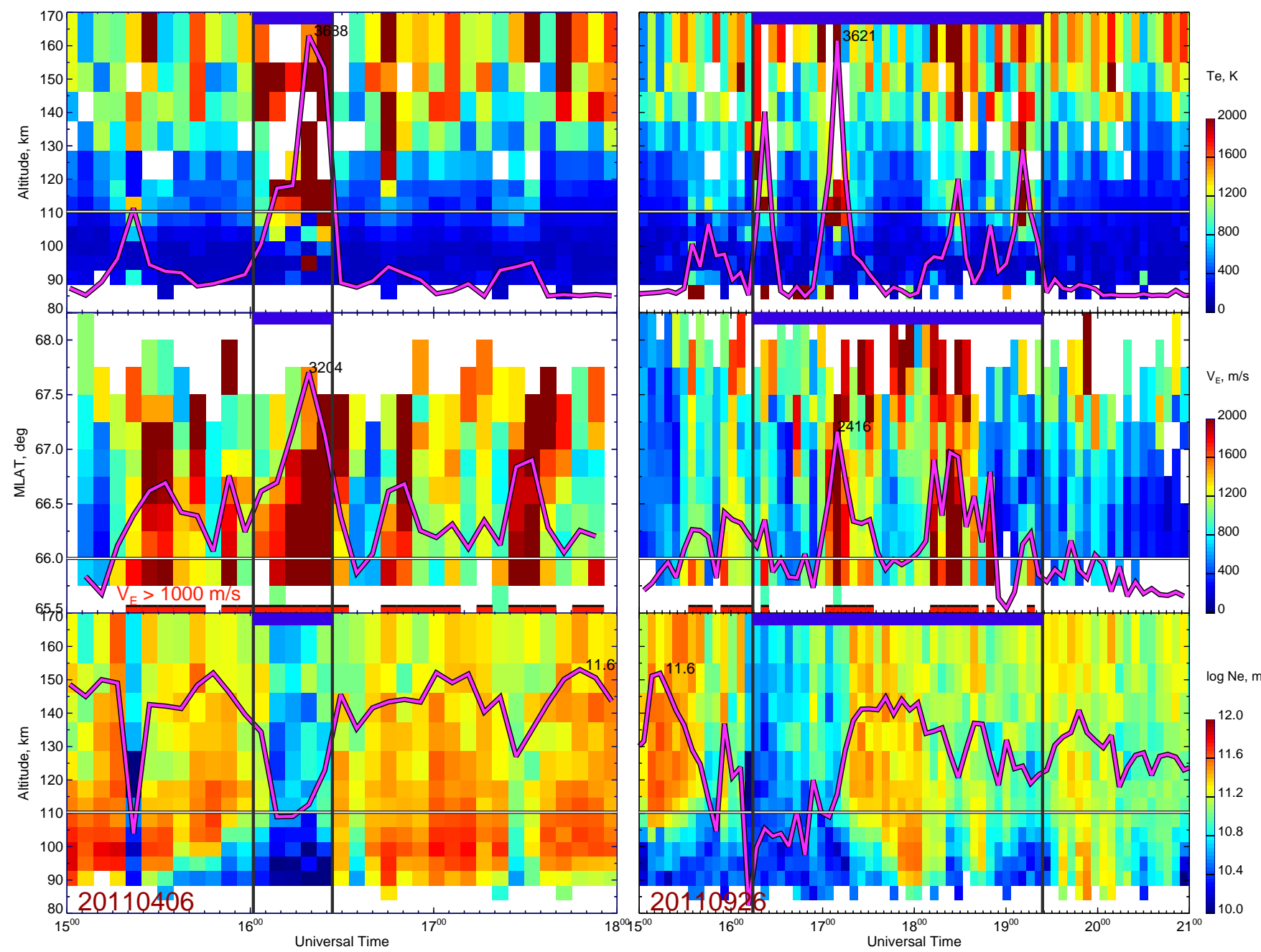

Fig. 3. Examples of anomalous electron heating (AEH) events. The three rows represent (from top to bottom): $\mathrm{AC}$ electron temperatures in the field-aligned beam 64157 versus UT and altitude, LP ion velocity for various MLAT bins, and AC electron density in beam 64157. The colour bars are shown to the right of each row. The black-white line shows an altitude of $\sim 110 \mathrm{~km}$ for the $T_{\mathrm{e}}$ and $N_{\mathrm{e}}$ datasets and a MLAT of $66.0^{\circ}$ for the $V_{E}$ dataset. The pink line shows time variation of the corresponding parameter at the bin shown by the black-white line in arbitrary scale. The maximum parameter value is shown by the digits near the peak. The dark red horizontal lines at the bottom of the middle row show intervals with $V_{E}>1000 \mathrm{~ms}^{-1}$. Black vertical lines and colour stripes show the AEH intervals.

This is further investigated in Fig. 5 that shows occurrence probabilities of (a) high convection velocity at MLAT $=66^{\circ}$, (b) high electron temperature at $110 \mathrm{~km}$ in beam 64157 , and (c) AEH hourly intervals versus MLT. The occurrence probabilities of high $V_{E}$ and $T_{\mathrm{e}}$ were estimated by counting all PFISR measurements in 2011 that were above a certain lower limit for each hourly bin and dividing by the total number of measurements in that MLT bin. Similarly, the AEH probability was estimated as the number of AEH hourly intervals in 2010-2011 divided by the number of hourly intervals covered by PFISR. The overall AEH occurrence probability was also estimated and for this combined dataset it was $0.3 \%$ (dotted line in Fig. 5c), which agrees well with the more rigorous estimates given in Fig. $4 \mathrm{c}$ and d.
As discussed above, one expects a reasonable match between periods of high $V_{E}$ and $T_{\mathrm{e}}$ in terms of their diurnal variations and typical probability values and this is what is observed in the first two panels, where the red histograms show very similar values and variations with MLT. The information about $V_{E}$ distribution and occurrence of high $V_{E}$ measurements is important not only for the current study but also for the $\mathrm{E}$ region irregularity studies in general. This is because electric fields in excess of $20-30 \mathrm{mV} \mathrm{m}^{-1}\left(V_{E}=\right.$ $400-500 \mathrm{~m} \mathrm{~s}^{-1}$ ) are required for generation of the primary MTSI waves and these estimates can be therefore used as a first-order approximation for the occurrence of irregularities generated directly by the MTSI. This information is rarely available and nearly-continuous PFISR observations provide 

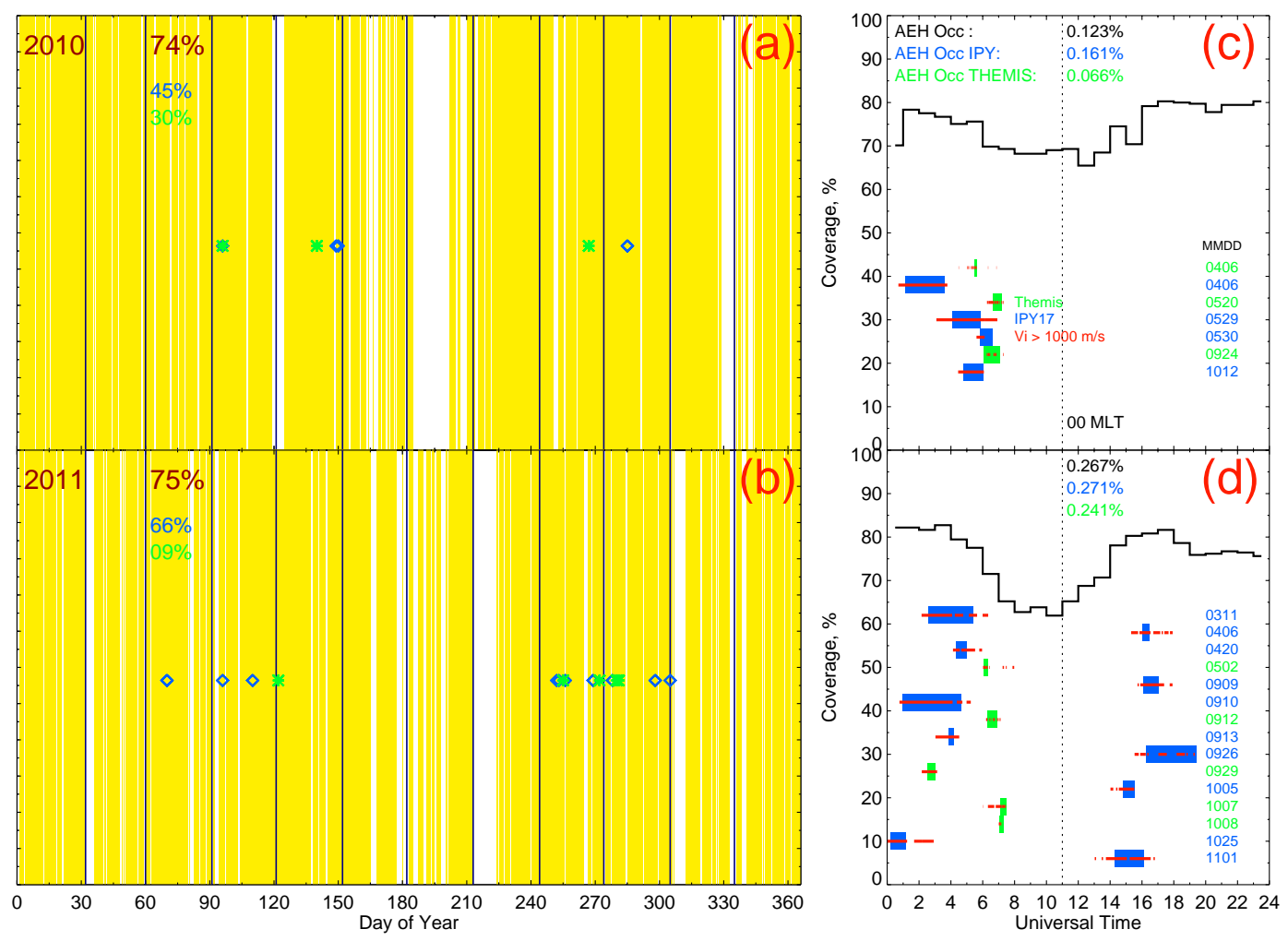

Fig. 4. PFISR coverage in 2010-2011 versus (a) and (b) time from start of the year and (c) and (d) universal time. The vertical dashed line in (c) and (d) shows magnetic midnight. The yellow areas in (a) and (b) show PFISR operating in the IPY or THEMIS mode. The diamonds (IPY) and asterisks (THEMIS) in the middle show identified AEH events. The percentages in (a) and (b) represent total coverage by both modes combined (top, red), by the IPY mode (blue, middle), and by the THEMIS mode (green, bottom). In panels (c) and (d), the colour stripes show the AEH intervals in UT, while the red lines show intervals of elevated $V_{E}>1000 \mathrm{~ms}^{-1}$. The AEH events in the MMDD format are given at the right of panels (c) and (d). The percentages in (c) and (d) show occurrence probability of AEH events for both modes combined and for each of the two modes.

important context for predicting when these irregularities are expected.

Comparing occurrences of high $V_{E}$ and $T_{\mathrm{e}}$ with that of AEH events, one can notice a general agreement in terms of their diurnal variations, i.e. AEH events were observed near the peaks of high $V_{E}$ occurrence, as expected. One important exception was however, that no AEH events were observed on both sides of 00:00 MLT. Occurrence of both high $V_{E}$ and $T_{\mathrm{e}}$ was reduced in this time sector, but certainly not to the same extent as the AEH occurrence was. The more striking difference was that the AEH occurrence was at least one order of magnitude smaller than relevant occurrences of PFISR measurements with $V_{E}>1000 \mathrm{~m} \mathrm{~s}^{-1}$ or $T_{\mathrm{e}}>800 \mathrm{~K}$. The possible reasons for these discrepancies are discussed in Sect. 6.1.

\section{Multi-point observations of electron heating events}

In analysing the electron temperature dependence on the convection electric field it is essential to achieve the best possible spatial match between these two types of measurements. By employing multi-beam observations with PFISR it is possible to achieve this by matching the AC range gate with the closest MLAT bin. Analyses of matched observations in multiple points would then provide a useful 2-D context for AEH investigation, and in this section the results of this analysis are presented.

Figure 1 shows that different PFISR beams have different nominal MLAT bin matches with the AC range gate of $110 \mathrm{~km}$. To investigate whether these nominal bins were also the bins with largest $T_{\mathrm{e}}-E$ correlation, the rank correlation coefficients were calculated for each event between $T_{\mathrm{e}}$ measurements in each beam at $110 \mathrm{~km}$ and $E$ measurements at various MLAT bins. The data in the first two MLAT bins were not considered here as those bins had large errors in $E$ because of the pointing geometry of ion drift velocity vectors that fall into those bins. The correlation analysis (not shown here for brevity) generally confirmed that the nominallymatched MLAT bin from experiment geometry was also the MLAT bin with the best $T_{\mathrm{e}}-E$ correlation. In the following analyses of the $T_{\mathrm{e}}$-vs.- $E$ relationship we therefore used the $E$ data from these MLAT bins only. 


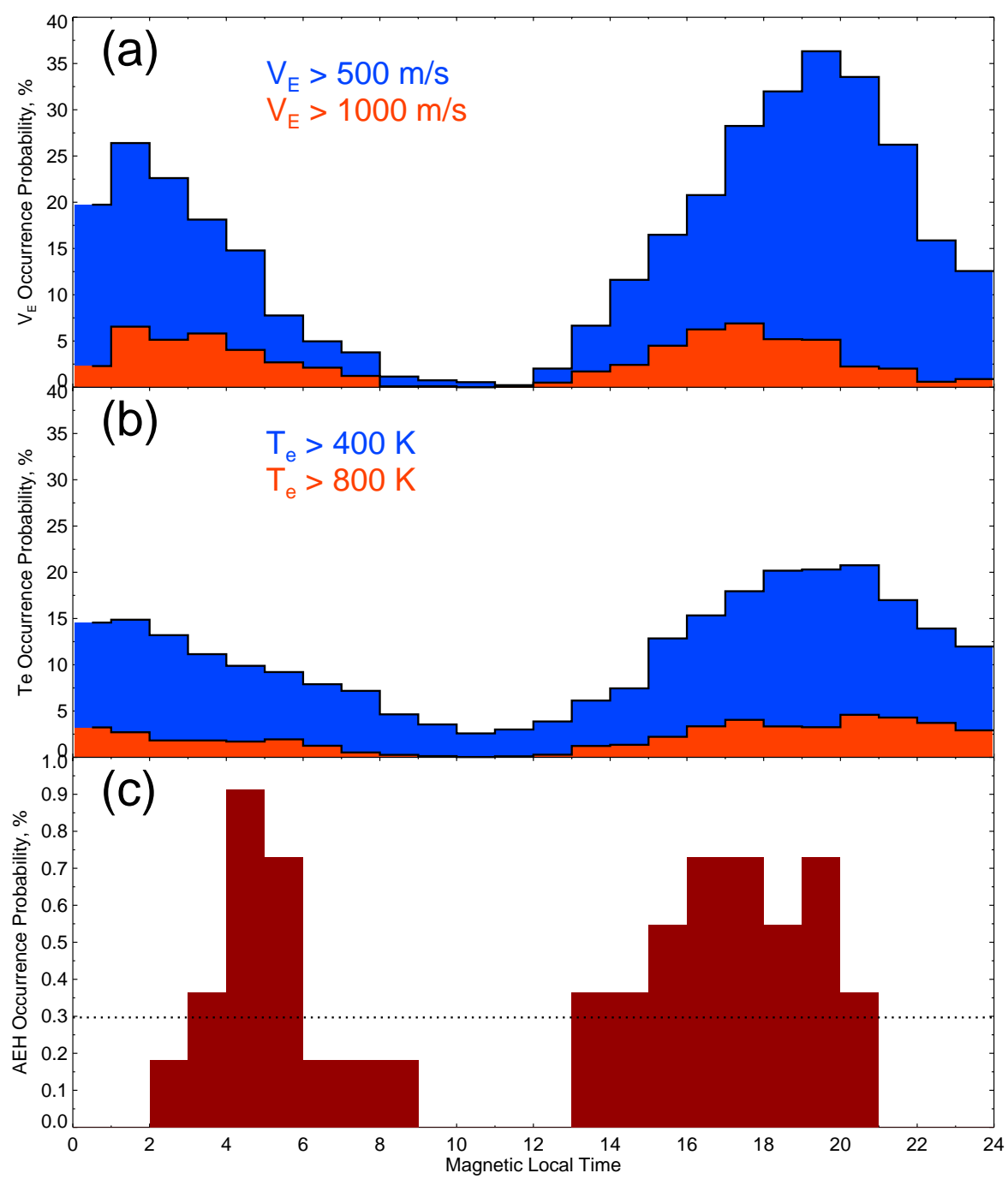

Fig. 5. Occurrence probabilities versus MLT for (a) high convection velocity $V_{E}$, (b) high electron temperature $T_{\mathrm{e}}$, and (c) AEH hourly intervals. The overall AEH probability of $0.3 \%$ is marked by a dotted line in panel (c).

Figure $6 \mathrm{a}$ and $\mathrm{b}$ presents the measured relationship between $T_{\mathrm{e}}$ and $E$ in the IPY mode in 2011 for beam 65066 (azimuth $75.03^{\circ} \mathrm{N}$, elevation $65.56^{\circ}$; Fig. 1). Out of 3 datasets for different modes, this was the most extensive dataset with $10 \mathrm{AEH}$ events and 1560 points including 35 points with extreme fields $E>120 \mathrm{mVm}^{-1}$. Out of 4 IPY beams, this beam had the largest $T_{\mathrm{e}}-E$ correlation ( 0.76 for all points and 0.6 for $E>40 \mathrm{mVm}^{-1}$ ). As mentioned in Sect. 2, both the AC data on $T_{\mathrm{e}}$ and $N_{\mathrm{e}}$ and LP electric fields were obtained from re-analysed AEH dataset at 2 min time resolution. Following Williams et al. (1992), all points with electron densities below $3 \times 10^{10} \mathrm{~m}^{-3}$ were excluded. In addition, points with very large $T_{\mathrm{e}}$ uncertainties were excluded $\left(\delta T_{\mathrm{e}}>\right.$ $1000 \mathrm{~K} ; \delta T_{\mathrm{e}} / T_{\mathrm{e}}>100 \%$ ). Figure $6 \mathrm{a}$ also presents information about the electron density by the colour-coding, while Fig. $6 \mathrm{~b}$ shows the data binned in increments $E=10 \mathrm{mV} \mathrm{m}^{-1}$. The grey circles (vertical bars) in Fig. $6 \mathrm{~b}$ show the mean (standard deviation) values for each bin. The 3 model dependencies from Fig. 2 are also given, as well as the current model results at $108 \mathrm{~km}$. Figures $6 \mathrm{c}$ and d present fractions of points that were, within uncertainty, in agreement with (green), below (blue), or above (red) the model trend for two of these models.

The majority of points lie in the sector roughly centred at the expected linear trend shown by the dotted line, Fig. $6 \mathrm{a}$. There is no clear pattern in points based on their background electron density represented by the colour, although one can perhaps see that many points with the lowest densities (blue) are located farther away from the model trends. If the above limitation of $N_{\mathrm{e}}>3 \times 10^{10} \mathrm{~m}^{-3}$ is removed, then even more points appear in these areas away from the trends, which may imply that higher $N_{\mathrm{e}}$ threshold is needed in some cases. Since results of this analysis conducted with a higher density 

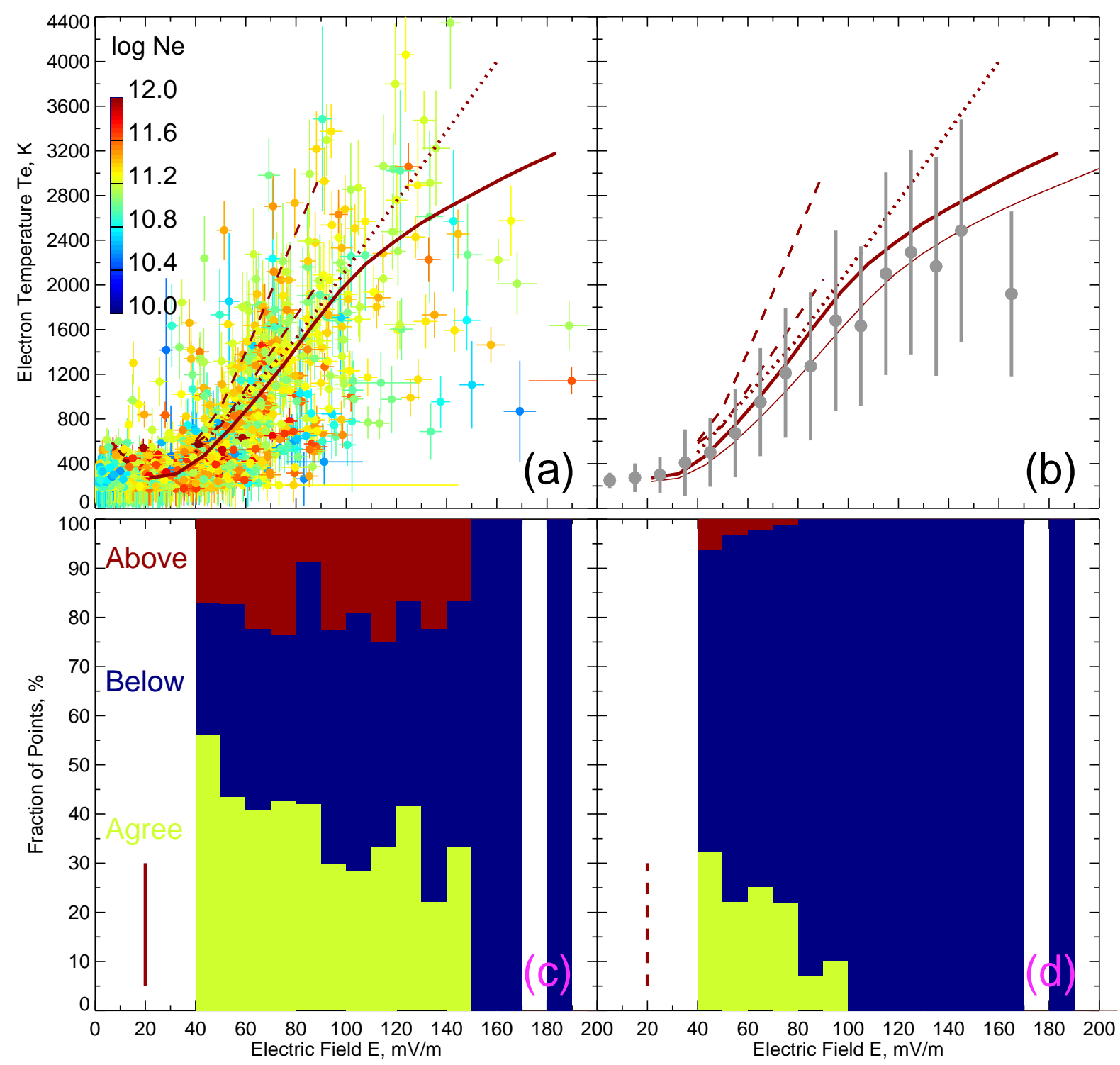

Fig. 6. (a) and (b) Electron temperature $T_{\mathrm{e}}$ versus electric field $E$ in beam 65066 for all AEH events identified in the IPY mode dataset in 2011. In panel (a), all individual points are colour-coded in the electron density $N_{\mathrm{e}}$ in the same range gate as $T_{\mathrm{e}}$, while in panel (b) the grey circles and bars show the binned data at $10 \mathrm{mV} \mathrm{m}^{-1}$ increments. The selected model dependencies are given by the dark red lines (the same models as in Fig. 2b). In addition, the current model results at $108 \mathrm{~km}$ are given by the thin line. (c) and (d) The green bars in the bottom panels show the fraction of points in agreement, within uncertainties, with the (c) solid and (d) upper dashed model trends. The blue (red) bars also show fraction of points below (above) the trend.

threshold were largely unchanged and for consistency with previous studies, the limit $N_{\mathrm{e}}>3 \times 10^{10} \mathrm{~m}^{-3}$ was left as is.

The grey binned trend in Fig. $6 \mathrm{~b}$ is in general agreement, within uncertainty, with both dotted and solid trends. One can notice however, that the average binned values represented by the grey circles are more consistent with the solid trend. Moreover, the overall shape of the $T_{\mathrm{e}}$-vs.- $E$ dependence represented by the grey circles is not linear and more similar to that of the solid trend. This is due to points at high electric fields $E>100 \mathrm{mV} \mathrm{m}^{-1}$ being, on average, lower by
$500-1000 \mathrm{~K}$ than the linear trend. While for binned points at $E=100-130 \mathrm{mV} \mathrm{m}^{-1}$ this is still within their uncertainty, this is not so at the 3 highest bins with significant number of points which are all below the linear trend, within uncertainty. The two of these points at $E=130-150 \mathrm{mV} \mathrm{m}^{-1}$ are consistent with the solid trend, while the rightmost point at $E=160-170 \mathrm{mV} \mathrm{m}^{-1}$ is below both dotted and solid trends, Fig. 6b. Finally, the same model results but at $108 \mathrm{~km}$ appear to match the binned measurements at high electric fields even better; this is further discussed in Sect. 6.2. 
The fraction of points consistent with the solid trend shows a general decrease with an increasing $E$, Fig. 6c, so that at large electric fields $E>80 \mathrm{mV} \mathrm{m}^{-1}$ points below the trend start to dominate; blue bars are the highest and in many cases $>50 \%$. This feature becomes even more pronounced if the same analysis is conducted for the linear dotted trend (not presented here for brevity). This feature is also most obvious at the highest electric fields $E>150 \mathrm{mV} \mathrm{m}^{-1}$ in Fig. 6c. Even though the number of points at these very high values is low, the uncertainties are small enough to conclude that all points at these extreme electric fields are below the trend within uncertainty, Fig. 6c.

Finally, Fig. 6 also shows that the upper dashed line representing non-Maxwellian case in the model by Milikh and Dimant (2003) appears to bound the points from above, because only $\sim 5 \%$ of points are above the upper dashed line at $E=40-80 \mathrm{mV} \mathrm{m}^{-1}$, within uncertainty, and all points are below it at $E>100 \mathrm{mV} \mathrm{m}^{-1}$, Fig. 6 d. This suggests that the model accounting for non-Maxwellian deviations in the electron energy distribution can be regarded as an absolute upper limit on the electron temperature for a given electric field.

\section{Discussion}

In this study, the anomalous electron heating processes in the auroral E region were re-examined using an extensive 2year dataset collected with the new-generation ISR system at Poker Flat, Alaska. The quasi-continuous nature of PFISR operations allowed us, for the first time, to estimate the overall AEH occurrence probability and address the fundamental question of what local conditions are conducive to AEH occurrence. The fundamental dependence of the electron temperature on the convection electric field was re-examined focusing on departures from the expected linear trend. Below the two groups of issues are discussed in relation to the major objectives of the current study.

\subsection{Anomalous electron heating occurrence}

This study employed the PFISR data collected in 20102011 and developed an automatic algorithm to identify AEH events as described in Sect. 4. To keep computational requirements at a manageable level, in searching for $\mathrm{AEH}$ events we focused on the electron temperature observations in the magnetic-field-aligned beam 64157, Fig. 1, and at the range gate nearest to a $110 \mathrm{~km}$ altitude, where strongest AEH effects are expected both from past observations and models (e.g. Schlegel and St.-Maurice, 1981; Williams et al., 1992; Robinson and Honary, 1993). Once the AEH events were found however, the entire range of PFISR data products was employed for further analysis including data collected in other PFISR beams and other $\mathrm{E}$ region range gates. The developed set of criteria for AEH identification resulted in 22 unique events which were fully consistent with the previously-found common features of AEH events: (1) strong electron temperature enhancements peaking near $110 \mathrm{~km}$, but extending over several range gates, (2) electric field enhancements near the nominally-coincident MLAT bin, and (3) F region ion temperature enhancements. We are confident therefore, that the identified AEH events were a result of the true electron heating processes rather than a collection of random strong perturbations in the electron temperature in one location.

In studying AEH processes it is important to consider the magnetic latitudes of observations. In the polar E region, the electron temperature enhancements are primarily caused by the MTSI wave heating under conditions of strong electric fields with little contribution from particle precipitation. Such events are observed during geomagnetic storms when electric fields are enhanced over extended periods (Milikh et al., 2006). In the auroral region, some electron heating is caused by precipitating particles and one expects AEH events to be more transient (Williams et al., 1992), i.e. to occur as (1) isolated bursts or (2) their sequences. The results of the current study were consistent with this expectation as all 22 events fell into these 2 major categories: an isolated burst was shown in Fig. 3 (left) and a sequence of bursts was presented in Fig. 3 (right).

The AEH events were found to occur predominantly near equinoxes and near 06:00 and 18:00 MLT, Figs. 4 and 5. One can attribute the first finding to the Russell-McPherron effect and elevated geomagnetic activity near equinoxes (Russell and McPherron, 1973). One would expect however, some geomagnetic activity away from equinoxes, albeit at reduced occurrence levels, and in this sense the total absence of AEH events in summer or winter is not easy to interpret. In terms of MLT occurrence trends, the total absence of AEH events near magnetic midnight and the fact that all events were observed either earlier than the peak in substorm occurrence of 22:00 MLT or much later indicates that substorms were not the primary geophysical drivers of AEH.

The absence of AEH events in the noon sector was consistent with the lack of high- $E$ and high- $T_{\mathrm{e}}$ events based on the PFISR data statistics, Fig. 5. This is most likely due to the auroral zone (within which high- $E$ events normally occur) positioned poleward of MLAT $=65^{\circ}$ in this MLT sector. In contrast, occurrence of high- $E$ and high- $T_{\mathrm{e}}$ events was greater near 00:00 MLT, Fig. 5. In particular, occurrence of high- $T_{\mathrm{e}}$ events only showed a small decrease near 00:00 MLT. From this one would expect at least a few AEH events in the midnight sector. The complete lack of such events observed in the current study may imply that high- $E$ events in this sector, while still present, are too short-lived to cause any sustained $T_{\mathrm{e}}$ increase.

The overall AEH occurrence probability was found to be low, $\sim 0.3 \%$, although it increased from $0.12 \%$ in 2010 to $0.27 \%$ in 2011 . We have also run the same search algorithm in 2009 and found no AEH events. These two observations suggest an increase with the solar activity. The hourly AEH 
occurrence was found to be substantially higher in the dawn and dusk sectors, Fig. 5c, which was consistent with statistics of high- $E$ and high- $T_{\mathrm{e}}$ events, Fig. 5. However, the AEH occurrence was at least one order of magnitude smaller than relevant occurrences of high- $E$ and high- $T_{\mathrm{e}}$ events, Fig. 5. The low overall occurrence indicates that AEH remains a relatively rare event. Overall, the results of AEH occurrence analysis suggested that elevated electric field, by itself, is not a sufficient condition.

The low AEH occurrence probability prompts an important question of whether specifics of the PFISR data fitting could have affected the occurrence analysis results. The two relevant factors in this context are the fitting model and integration period. One complication is that the standard analysis assumes $T_{\mathrm{e}}=T_{\mathrm{i}}$ below $\sim 114 \mathrm{~km}$ altitude. This is not a problem for a reasonably limited subset of data that can be reanalysed without this assumption (as was done in the second part of the current study). It is not feasible however, to do this for the entire dataset and one has to rely on the standard data products. To address this issue, we have manually examined daily $T_{\mathrm{e}}$ plots near equinoxes in 2011 looking for enhanced $T_{\mathrm{e}}$ between $100-120 \mathrm{~km}$ rather than just at $110 \mathrm{~km}$. No additional AEH events were found, which strongly suggests that the employed dataset and approach yielded the bulk of (if not all) AEH events that occurred within the period of interest.

In developing the AEH search criteria, we have also ensured that the PFISR datasets at all time resolutions resulted in true AEH events that all had the same morphology (in terms of the $T_{\mathrm{e}}, T_{\mathrm{i}}$ and $E$ variations). In combination with the fact that AEH occurrences in MLT were very similar for the THEMIS and IPY datasets in the pre-midnight sector (when the THEMIS mode was run), this indicates that different time integrations were not a major factor in finding AEH events. In addition, for one THEMIS event (20 May 2010), the data has also been post-integrated with a longer period of $15 \mathrm{~min}$ (the same as for the IPY mode in 2010) and the search algorithm has identified this AEH event with only a small difference in start and end times. This provides additional support to the above assertion that time integration had little effect on occurrence trends.

Finally, the "large-scale" AEH events analysed in the current study is what has traditionally attracted much of the research interest, particularly using ground-based observations of the E region as was described in Sect. 1. However, it is very likely that the AEH phenomena also include more local heating processes operating at scales smaller than a typical radar cell. As such the AEH occurrence probability analysed in the current study is likely to be an underestimate since it refers to a relatively narrow part of a broader spectrum of the AEH phenomena.

\subsection{Relationship between electron temperature and electric field}

According to most models, the convection drift velocity $V_{E}=1000 \mathrm{~m} \mathrm{~s}^{-1}$ would result in the electron temperature $T_{\mathrm{e}}$ increase to $\sim 800 \mathrm{~K}$ (at $110 \mathrm{~km}$ ), Fig. $2 \mathrm{~b}$. One can expect therefore, periods with $T_{\mathrm{e}}>800 \mathrm{~K}$ (a condition used for AEH search) to occur at the same time as periods with $V_{E}>1000 \mathrm{~m} \mathrm{~s}^{-1}$. An interesting finding from analysis of individual events was however, that AEH events were observed within the periods with $V_{E}>1000 \mathrm{~ms}^{-1}$, Figs. 3 and 4, but not matching them exactly or even approximately. Similarly, occurrence of events with $V_{E}>1000 \mathrm{~m} \mathrm{~s}^{-1}$ was much larger than AEH occurrence, Fig. 5. One possible interpretation for these features is that, in addition to enhanced electric field, some other condition(s) must be satisfied for the electron temperature to increase in line with the expected linear trend.

The results of our modelling analysis showed that some reduction in the rate of temperature increase with the drift velocity is expected, as compared with previous studies. First, the model heating rates depend on the ratio of collision frequency and gyrofrequency, with the latter depending on the magnetic field magnitude $B$ and hence MLAT of observations. The differences in the model electron temperatures due to changes in MLAT and $B$ were estimated to be $\sim 100 \mathrm{~K}$, Fig. 2b. The second and more significant effect occurs at very large drift values $V_{E}>1800 \mathrm{~m} \mathrm{~s}^{-1}$, Fig. 2b. Above this value, the model predicted a somewhat weaker AEH effect, with the solid curve deviating from the linear trend in Fig. 2b, due to a much stronger vibrational cooling, Fig. 2a.

Implicit in the current AEH model was a wave-induced increase in the anomalous collision frequency $v_{\mathrm{e}}^{*}$ (Sudan, 1983; Robinson, 1986). Although this quasi-linear approach has been largely successful in explaining radar observations of the $\mathrm{E}$ region electron temperatures and irregularity phase velocities, a complete, self-consistent description of the nonlinearly-saturated MTSI turbulence, particularly at high electric fields, is still lacking (see, e.g. discussion in Dimant and Milikh, 2003). One should therefore, bear in mind that none of the existing AEH models employ this self-consistent MTSI theory, although some promising results have been obtained using a heuristic model of turbulence (Dimant and Milikh, 2003; Milikh et al., 2006; Dimant and Oppenheim, 2011a,b; Oppenheim and Dimant, 2013).

The fundamental $T_{\mathrm{e}}$-vs.- $E$ relationship measured with the new-generation ISR system at Poker Flat was found to be very similar to those established with other ISR facilities. In the current study, these previously-measured relationships were represented by a dotted linear trend taken from the most recent study on the issue by Bahcivan (2007). This trend also matched really well the representative model trend taken from Robinson and Honary (1993). The PFISR-measured relationship was found to be linear between $40-100 \mathrm{mV} \mathrm{m}^{-1}$ and in close agreement with the linear trend represented by the dotted line in Fig. $6 \mathrm{~b}$. The average $T_{\mathrm{e}}$ values were 
$\sim 100 \mathrm{~K}$ smaller than those given by the linear trend, but this was likely to be due to the above-mentioned decrease in the heating rate with an increasing magnetic field $B$. The general consistency between the PFISR and other observations of AEH at moderate electric fields provided additional and independent validation of PFISR measurements, particularly of its electron temperature and electric field products. By the same token, the presented PFISR observations of AEH events strengthened conclusions derived from previouslyreported observations, while also pointing towards potentially important differences as discussed below.

At larger values $E>100 \mathrm{mVm}^{-1}$, the differences between the measured $T_{\mathrm{e}}$ and the linear trend were much more pronounced. At these $E$ values, the binned $T_{\mathrm{e}}$ values in Fig. $6 \mathrm{~b}$ were, on average, below the linear trend by $500-1000 \mathrm{~K}$. At the highest available electric fields $E>$ $150 \mathrm{mV} \mathrm{m}^{-1}$, both the binned $T_{\mathrm{e}}$ values and individual points were all below the linear trend within uncertainty, Fig. 6a-c.

As noted above, the AEH model extended to extreme electric fields showed a considerable change in the rate of $T_{\mathrm{e}}$ increase with $V_{E}$ at a drift value $V_{E} \approx 1800 \mathrm{~m} \mathrm{~s}^{-1}$, Fig. 2b. At this point, the empirical vibrational cooling starts to increase more sharply with $T_{\mathrm{e}}$, Fig. 2a, and this may help explain at least some of the deviation from the linear trend at extreme electric fields.

A possibly related feature was that the general shape of the measured $T_{\mathrm{e}}$-vs. $-E$ relationship was quite similar to that given by the solid model trend, Fig. $6 \mathrm{~b}$. This may indicate two possibilities. First, the neutral densities were higher than those used in our modelling calculations, even after corrections described in Sect. 3. Second, the altitude where maximum AEH effects were observed was not $110 \mathrm{~km}$, but 1$2 \mathrm{~km}$ lower. Unfortunately, the range resolution of PFISR is limited by its pulse length and for the considered data products and observational conditions the practical lower limit was $4.5 \mathrm{~km}$. This makes it impossible to directly confirm the second assertion by using PFISR observations. Nevertheless, additional modelling results for an altitude of $108 \mathrm{~km}$ showed a much better agreement with observations, Fig. 6 b. One should also note here that in the considered beam 65066 the altitude gate was actually $108.6-113.1 \mathrm{~km}$, which implies that there is an uncertainty of the same order $1-2 \mathrm{~km}$ in matching modelling calculations and observations.

At the largest available electric fields $E>150 \mathrm{mV} \mathrm{m}^{-1}$, all points were below both dotted and solid trends in Fig. 6 . As noted earlier, there were only a few points at these extreme $E$ values so one has to be careful in interpreting this limited subset of data. Nevertheless, these measurements do not appear to be much different from other, more numerous points nearby at $E=120-140 \mathrm{mV} \mathrm{m}^{-1}$ and this suggests that these were valid measurements. Further analysis showed that similar high- $E$, low- $T_{\mathrm{e}}$ points were also present in other beams and MLAT bins, which also points towards their reasonable quality. It is expected that continuous operations of PFISR on the ascending phase of the solar cycle 24 will yield more AEH events, including observations at extreme electric fields, which will allow to further verify the presented results.

\section{Summary and conclusions}

Statistical analysis of the anomalous electron heating (AEH) processes observed by PFISR in the auroral E region showed that:

1. The overall occurrence of AEH events observed with PFISR is below $1 \%$, which suggests that a number of conditions must be satisfied for radar signatures of AEH to be observed. Diurnal variation of AEH events is broadly consistent with occurrences of high-electricfield and high-electron-temperature observations, except for much smaller AEH occurrence and lack of AEH events near magnetic midnight. Presence of strong electric fields therefore, appears to be a necessary condition for AEH, but not sufficient. The best chance to observe $\mathrm{AEH}$ in the auroral region is near equinoxes and in the dusk and dawn sectors, away from typical times of substorms. The strongest AEH events occur between auroral precipitation regions.

2. The relationship between the electron temperature and convection electric field measured with PFISR is mostly linear and consistent with previously-reported observations at moderate electric fields $E=40-100 \mathrm{mV} \mathrm{m}^{-1}$. At higher electric fields $E>100 \mathrm{mV} \mathrm{m}^{-1}$, the electron temperatures are lower than expected from extrapolating the previously-obtained linear trend, possibly an indication of AEH saturation. Some of this deviation from the linear trend may be due to a larger increase in vibrational cooling at very large temperatures and electric fields. An absolute upper limit on the electron temperature for a given electric field may be associated with non-Maxwellian distribution of the electron energy.

Acknowledgements. PFISR is operated by SRI International under NSF Cooperative Agreement AGS-1133009. This research was supported by NSF grants ANT-1139806 and AGS-1243476.

Topical Editor K. Hosokawa thanks Y. Dimant and one anonymous referee for their help in evaluating this paper.

\section{References}

Bahcivan, H.: Plasma wave heating during extreme electric fields in the high-latitude E region, Geophys. Res. Lett., 34, L15106, doi:10.1029/2006GL029236, 2007.

Bahcivan, H., Cosgrove, R. B., and Tsunoda, R. T.: Parallel electron streaming in the high-latitude $\mathrm{E}$ region and its effect on the incoherent scatter spectrum, J. Geophys. Res., 111, A07306, doi:10.1029/2005JA011595, 2006. 
Dimant, Y. S. and Milikh, G. M.: Model of anomalous electron heating in the E region: 1. Basic theory, J. Geophys. Res., 108, 1350, doi:10.1029/2002JA009524, 2003.

Dimant, Y. S. and Oppenheim, M. M.: Magnetosphere-ionosphere coupling through E region turbulence: 1. Energy budget, J. Geophys. Res., 116, A09303, doi:10.1029/2011JA016648, 2011a.

Dimant, Y. S. and Oppenheim, M. M.: Magnetosphere-ionosphere coupling through $\mathrm{E}$ region turbulence: 2 . Anomalous conductivities and frictional heating, J. Geophys. Res., 116, A09304, doi:10.1029/2011JA016649, 2011b.

Fejer, B. G. and Kelley, M. C.: Ionospheric irregularities, Geophys. Rev., 18, 401-454, 1980.

Foster, J. C. and Erickson, P. J.: Simultaneous observations of Eregion coherent backscatter and electric field amplitude at Fregion heights with the Millstone Hill UHF Radar, Geophys. Res. Lett., 27, 3177-3180, 2000.

Hedin, A. E.: Extension of the MSIS thermospheric model into the middle and lower Atmosphere, J. Geophys. Res., 96, 1159-1172, 1991.

Heinselman, C. J. and Nicolls, M. J.: A Bayesian approach to electric field and E-region neutral wind estimation with the Poker Flat Advanced Modular Incoherent Scatter Radar, Radio Sci., 43, RS5013, doi:10.1029/2007RS003805, 2008.

Igarashi, K. and Schlegel, K.: Electron temperature enhancements in the polar E-region measured with EISCAT, J. Atmos. Terr. Phys., 49, 273-280, 1987.

Janhunen, P.: Perpendicular particle simulation of the E region Farley-Buneman instability, J. Geophys. Res., 99, 11461-11474, 1994.

Lyons, L. R., Zou, S., Heinselman, C. J., Nicolls, M. J., and Anderson, P. C.: Poker Flat radar observations of the magnetosphereionosphere coupling electrodynamics of the earthward penetrating plasma sheet following convection enhancements, J. Atmos. Sol. Terr. Phys., 71, 717-728, 2009.

Makarevich, R. A.: HF radar observations of high-velocity E-region echoes from the eastward auroral electrojet, J. Geophys. Res., 113, A09321, doi:10.1029/2008JA013204, 2008.

Milikh, G. M. and Dimant, Y. S.: Model of anomalous electron heating in the E region: 2. Detailed numerical modeling, J. Geophys. Res., 108, 1351, doi:10.1029/2002JA009527, 2003.

Milikh, G. M., Goncharenko, L. P., Dimant, Y. S., Thayer, J. P., and McCready, M. A.: Anomalous electron heating and its effect on the electron density in the auroral electrojet, Geophys. Res. Lett., 33, L13809, doi:10.1029/2006GL026530, 2006.

Nielsen, E. and Schlegel, K.: Coherent radar Doppler measurements and their relationship to the ionospheric electron drift velocity, J. Geophys. Res., 90, 3498-3504, 1985.

Oppenheim, M. M. and Dimant, Y. S.: Kinetic simulations of 3-D Farley-Buneman turbulence and anomalous electron heating, J. Geophys. Res., 118, 1306-1318, doi:10.1002/jgra.50196, 2013.

Robinson, T. R.: Towards a self-consistent nonlinear theory of radar aurora backscatter, J. Atmos. Terr. Phys., 48, 417-422, 1986.

Robinson, T. R. and Honary, F.: A resonance broadening kinetic theory of the modified two-stream instability: Implications for radar auroral backscatter experiments, J. Geophys. Res., 95, 10731085, 1990.
Robinson, T. R. and Honary, F.: Adiabatic and isothermal ionacoustic speeds of stabilized Farley-Buneman waves in the auroral E-region, J. Atmos. Terr. Phys., 55, 65-77, 1993.

Russell, C. T. and McPherron, R. L.: Semi-annual variation of geomagnetic activity, J. Geophys. Res., 78, 92-108, 1973.

Schlegel, K.: Reduced effective recombination coefficient in the disturbed polar E-region, J. Atmos. Terr. Phys., 44, 183-185, 1982.

Schlegel, K. and St.-Maurice, J.-P.: Anomalous heating of the polar E region by unstable plasma waves. 1. Observations, J. Geophys. Res., 86, 1447-1452, 1981.

Schunk, R. W. and Nagy, A. F.: Electron temperatures in the F region of the ionosphere: Theory and observations, Rev. Geophys. Space Phys., 16, 355-399, 1978.

Schunk, R. W. and Nagy, A. F.: Ionospheres of the terrestrial planets, Rev. Geophys. Space Phys., 18, 813-852, 1980.

Semeter, J., Butler, T., Heinselman, C., Nicolls, M., Kelly, J., and Hampton, D.: Volumetric imaging of the auroral ionosphere: Initial results from PFISR, J. Atmos. Sol. Terr. Phys., 71, 738-743, 2009.

Sojka, J. J., Nicolls, M. J., Heinselman, C. J., and Kelly, J. D.: The PFISR IPY observations of ionospheric climate and weather, J. Atmos. Sol. Terr. Phys., 71, 771-785, 2009.

Stauning, P.: Absorption of cosmic noise in the E-region during electron heating events, Geophys. Res. Lett., 11, 1184-1187, 1984.

Stauning, P. and Olesen, J. K.: Observations of the unstable plasma in the disturbed polar E-region, Physica Scripta, 40, 325-332, 1989.

St.-Maurice, J.-P. and Laher, R.: Are observed broadband plasma wave amplitudes large enough to explain the enhanced electron temperatures of the high-latitude E region?, J. Geophys. Res., 90, 2843-2850, 1985.

St.-Maurice, J. P. and Schlegel, K.: Estimates of plasma wave amplitudes in the turbulent high-latitude E region using electron temperature measurements, J. Geophys. Res., 87, 5197-5201, 1982.

St.-Maurice, J.-P., Schlegel, K., and Banks, P. M.: Anomalous heating of the polar E region by unstable plasma waves. 2. Theory, J. Geophys. Res., 86, 1447-1452, 1981.

St.-Maurice, J.-P., Cussenot, C., and Kofman, W.: On the usefulness of $\mathrm{E}$ region electron temperatures and lower $\mathrm{F}$ region ion temperatures for the extraction of thermospheric parameters: a case study, Ann. Geophys., 17, 1182-1198, doi:10.1007/s00585999-1182-2, 1999.

Sudan, R. N.: Unified theory of type 1 and type 2 irregularities in the equatorial electrojet, J. Geophys. Res., 88, 4853-4860, 1983.

Wickwar, V. B., Lathuillere, C., Kofman, W., and Lejeune, G.: Elevated electron temperatures in the auroral $E$ layer measured with the Chatanika radar, J. Geophys. Res., 86, 4721-4730, 1981.

Williams, P. J. S., Jones, B., and Jones, G. O. L.: The measured relationship between electric field strength and electron temperature in the auroral E-region, J. Atmos. Terr. Phys., 54, 741-748, 1992. 